\title{
Evaluation of Different Thickness, Die Color, and Resin Cement Shade for Veneers of Multilayered CAD/CAM Blocks.
}

Çömlekoğlu, Muharrem Erhan ; Paken, Gamze ; Tan, Firuzan ; Dündar-Çömlekoğlu, Mine ; Özcan, Mutlu ; Akan, Ender ; Aladağ, Akın

\begin{abstract}
PURPOSE To investigate the effect of resin cement color on the post-cementation shade of thin laminate veneers milled from multilayered glass ceramic blocks for each layer. MATERIALS AND METHODS Thin slices (N $=180)$ with two thicknesses (A: $8 \times 8 \times 0.55 \mathrm{~mm}$ and B: $8 \times 8 \times 0.8 \mathrm{~mm} ; \mathrm{n}=90 /$ group $)$ were obtained from multilayered glass ceramic blocks using a diamond saw. Resin die specimens were prepared $(8 \times 8 \times 4 \mathrm{~mm})(\mathrm{n}=180)$ and divided into two resin cement colors (D: -3 . L: +3 ) and one control group (C: translucent). Each slice was luted to the composite specimens using a dual-curing resin cement under a constant load of $250 \mathrm{~g}$ and light-polymerized for 120 seconds. Specimens were placed on a white background and CIELab values for each layer of the blank slices (incisal [In], body [Bo], and cervical [Ce]) were recorded using a dental contact spectrophotometer. The values were calculated as $\triangle \mathrm{E}$, and the data were statistically analyzed (one-way ANOVA, Shapiro-Wilk). RESULTS Mean $\triangle \mathrm{E}$ values for $\mathrm{A}$ and $\mathrm{B}$ were significant $(\mathrm{p}<0.05)$ for In $(\mathrm{A}: 6.2 \pm 1.1$; B: $6.6 \pm 1.2)$, Bo (A: $5.1 \pm 2.6$; B: $3.9 \pm 1)$, and $\mathrm{Ce}(\mathrm{A}: 5.3 \pm 2.7 ; \mathrm{B}: 3.8 \pm 1.2)$. Mean $\triangle \mathrm{E}$ values for $\mathrm{In}$ for $\mathrm{D}$ and $\mathrm{L}$ for $\mathrm{A}$ and $\mathrm{B}$ groups were significantly higher $(6.3$ $\pm 1.2 ; 6.6 \pm 1.2)$ than Bo and Ce for $\mathrm{D}(4.4 \pm 2.4 ; 4.6 \pm 2.6)$ and $\mathrm{L}(4.6 \pm 1.4 ; 4.5 \pm 1.7)$ groups $(\mathrm{p}<0.05)$, respectively. Mean differences between Bo and Ce for $A$ and B groups were insignificant $(p \geq 0.05)$, while for In the differences were significant. Mean differences between Bo and Ce for A $(5.1 \pm 2.6 ; 5.3 \pm 2.7)$ and for B $(3.9 \pm 1 ; 3.5 \pm 1.2)$ groups were insignificant $(\mathrm{p} \geq 0.05)$, while for In the differences were significant $(6.2 \pm 1.1$ for $\mathrm{A}$ and $6.6 \pm 1.2$ for B). CONCLUSION The color changes for body and cervical regions of multilayered ceramic veneers were not affected by resin color or ceramic thickness, but the incisal area was affected. The translucency of each layer in ceramic veneer fabricated with multilayered blanks should be considered for clinical situations.
\end{abstract}

DOI: https://doi.org/10.1111/jopr.12367

Posted at the Zurich Open Repository and Archive, University of Zurich

ZORA URL: https://doi.org/10.5167/uzh-127840

Journal Article

Accepted Version

Originally published at:

Çömlekoğlu, Muharrem Erhan; Paken, Gamze; Tan, Firuzan; Dündar-Çömlekoğlu, Mine; Özcan, Mutlu; Akan, Ender; Aladağ, Akın (2016). Evaluation of Different Thickness, Die Color, and Resin Cement Shade for Veneers of Multilayered CAD/CAM Blocks. Journal of Prosthodontics, 25(7):563-569.

DOI: https://doi.org/10.1111/jopr.12367 


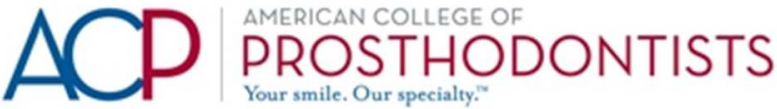

\section{Evaluation of Different Thickness, Die Colour and Resin Cement Shade for Veneers of Multilayered CAD/CAM Blocks}

\begin{tabular}{|r|l|}
\hline Journal: & Journal of Prosthodontics \\
\hline Manuscript ID: & JOPR-15-137 \\
\hline Wiley - Manuscript type: & Original Manuscript \\
\hline Index Words: & $\begin{array}{l}\text { Colour, Dental spectrophotometer, Multilayered CAD/CAM blocks, Resin } \\
\text { cement }\end{array}$ \\
\hline Manuscript Categories: & Basic Science \\
\hline \multicolumn{2}{|l}{} \\
\hline
\end{tabular}


Evaluation of Different Thickness, Die Colour and Resin Cement Shade for Veneers of Multilayered CAD/CAM Blocks

Running Title: Color Outcome of Luted Multilayered CAD/CAM Veneers

\title{
Keywords
}

Dental spectrophotometer, multilayered CAD/CAM blocks, colour, resin cement

\section{Correspondence}

The authors deny any conflict of interest.

\begin{abstract}
Purpose: To investigate the effect of resin cement color on the post-cementation shade of thin laminate veneers milled from multilayered glass ceramic blocks for each layer.

Materials and Methods: Thin slices $(\mathrm{N}=180)$ with 2 different thicknesses $(\mathrm{A}: 8 \times 8 \times 0,55 \mathrm{~mm}$ and B:8x8x0,8mm; n=90/group) were obtained from multilayered glass ceramic blocks (IPS Empress CAD C14 A2, IvoclarVivadent) using a diamond saw (Isomet 1000, Buehler). Resin die specimens were prepared (IPS Natural Die Material, B1, IvoclarVivadent) $(8 \times 8 \times 4 \mathrm{~mm})(\mathrm{n}=180)$ and divided into 2 different resin cement colours (D:-3, L:+3) and 1 control group (C:translucent) and each slice was luted to the composite specimens using a dual-curing resin cement (Variolink II, IvoclarVivadent) under a constant load of $250 \mathrm{~g}$ and light-polymerized for 120 s (Bluephase G2,
\end{abstract}


IvoclarVivadent). Specimens were placed on a white background and CIELab values for each layer of the blank slices [incisal (In), body (Bo) and cervical (Ce)] were recorded using a dental contact spectrophotometer (VITA Easyshade Advance, Vita Zahnfabrik). The values were calculated as $\Delta E$ and the data were statistically analyzed (One-way ANOVA, Shapiro-Wilk).

Results: Mean $\Delta \mathrm{E}$ values for A and B were significant for In (A:6,2 $\pm 1,1 ; B: 6,6 \pm 1,2)$ Bo $(\mathrm{A}: 5,1 \pm 2,6$; $\mathrm{B}: 3,9 \pm 1)$ and $\mathrm{Ce}(\mathrm{A}: 5,3 \pm 2,7 ; \mathrm{B}: 3,8 \pm 1,2)$, respectively. $(\mathrm{p}<0,05)$. Mean $\Delta \mathrm{E}$ values in $\mathrm{In}$ for $\mathrm{D}$ and $\mathrm{L}$ for $\mathrm{A}$ and $\mathrm{B}$ groups were significantly higher $(6,3 \pm 1,2 ; 6,6 \pm 1,2)$ than Bo and Ce for D $(4,4 \pm 2,4 ; 4,6 \pm 2,6)$ and $\mathrm{L}(4,6 \pm 1,4 ; 4,5 \pm 1,7)$ groups $(\mathrm{p}<0.05)$, respectively. Mean differences between $\mathrm{Bo}$ and $\mathrm{Ce}$ for $\mathrm{A}(5,1 \pm 2,6 ; 5,3 \pm 2,7)$ and for $\mathrm{B}(3,9 \pm 1 ; 3,5 \pm 1,2)$ groups were insignificant ( $\mathrm{P} \geq 0.05)$ while for In the differences were significant $(6,2 \pm 1,1$ for $A$ and $6,6 \pm 1,2$ for $B)$.

Conclusion: The color changes for body and cervical regions of multilayered ceramic veneers were not affected from resin color or ceramic thickness while only incisal area was affected. Translucency of each layer in ceramic veneer fabricated with multilayered blanks should be considered for clinical situations.

\section{Introduction}

New adhesive technologies and more resistant aesthetic materials are being developed through the rapid improvements in computer controlled and more standardized digital dental workflow. ${ }^{1,4}$ Optimized ceramic systems and resin cements brought up parallel to these technologies, facilitate the creation of restorations with optical properties similar to those of the natural tooth. ${ }^{2}$ Achievement of superior esthetics is not a concern with the increased light transmission of enhanced ceramics, especially for anterior restorations. ${ }^{3}$ 
Previously, clinicians used monochromatic blocks especially for posterior restorations, i.e. inlays/onlays. However, the fact of complex optical illusional phenomena in anterior esthetics cannot always be met with uni-coloured esthetic materials without the need for final characterization of a dental technician. In order to overcome such esthetic disadvantages of a monochromatic restoration, multicoloured ceramic blocks have been designed to create a threedimensional layered structure. These ceramic blocks offer a gradient of chroma from the cervical to the incisal areas that replicate dentin and enamel in the same block. ${ }^{5,6}$

Adhesively luted ceramic laminate veneers that allow for superior translucency and consist of $0,5 \mathrm{~mm}$ to $1,0 \mathrm{~mm}$-thick ceramic bonded to prepared or unprepared teeth with resin cement, are a conservative alternative to complete coverage restorations with predictable longevity. ${ }^{7,8}$ On the other hand, with the recently developed materials and fabrication techniques, $0.3 \mathrm{~mm}$ thick prepless anterior veneers are also possible. ${ }^{9}$ The resin cements are subjected to discoloration after polymerization beneath where the thinner and more translucent ceramic laminate restorations might be negatively affected. ${ }^{10}$ However, a clinically successful esthetic restoration should fulfill the correct colour matching. ${ }^{11}$ The optical behavior of a ceramic restoration is determined by the combination of tooth structure color, ceramic layer thickness, and cement color. ${ }^{8}$ Light-cured resins are frequently preferred to chemically cured or dual-cured resins because of their color stability, optical properties polymerization mode preferences for adhesive cementation of ceramic veneers. $^{10}$

Color can be determined either visually or with a device. However, visual evaluation has been deemed inconsistent and unreliable, while instrumental measurement provides objective values. ${ }^{12}$ Color measuring devices, i.e. spectrophotometers and colorimeters have become popular since they offer accuracy, standardization, and numerical color expression. ${ }^{13}$ 
Monochromatic blocks exhibit a homogeneous structure and the final color of a restoration would be the same for each layer after adhesive cementation; but multilayered blocks have multiple translucencies and no studies on this issue exist evaluating the final color differences between each layer after adhesive cementation. ${ }^{14}$ Therefore, the objective of this study was to investigate the effect of resin cement color for each layer on the post-cementation shade of thin laminate veneers milled from multilayered glass-ceramic blocks. The null hypothesis was that the final color differences of each layer of a CAD/CAM multilayered glass-ceramic block would be similar regardless of die, ceramic and/or cement color.

\section{Materials and methods}

\section{Fabrication of the specimens}

Thin slices $(\mathrm{N}=180)$ at two different thicknesses $(\mathrm{A}: 8 \times 8 \times 0,55 \mathrm{~mm}$ and $\mathrm{B}: 8 \times 8 \times 0,8 \mathrm{~mm} ; \mathrm{n}=90 /$ group $)$ were obtained from multilayered glass-ceramic blocks (IPS Empress CAD Multi C14 A2, IvoclarVivadent, Schaan, Liechtenstein) using a low-speed diamond saw (Isomet 1000, Buehler, Illinois, USA) under water cooling (Figure 1). One hundred and eighty specimens were prepared from a resin die material $(8 \mathrm{x} 8 \mathrm{x} 4 \mathrm{~mm}$; IPS Natural Die Material, A1 and A3,5 IvoclarVivadent, Schaan, Liechtenstein) simulating prepared tooth and divided into two different resin cement colours (D:-3, L:+3) and one control group (C: translucent) (Figure 2). All luting surfaces of the ceramic and resin samples were grinded with gradient silicon carbide abrasive papers of grit 400, 600 and 1200 (Leco VP 100, Leco Instrumente GmbH, Germany) for standardization.

\section{Cementation of ceramics with resin cements}

Before cementation, ceramic surfaces were treated with $4.9 \%$ hydrofluoric acid (IPS Etching Gel; IvoclarVivadent) for 60 seconds, water-rinsed and air-dried. The silane (Monobond Plus; 
IvoclarVivadent) was applied for 60 seconds to the ceramic and die material surfaces. A dual-curing resin cement (Variolink Veneer, IvoclarVivadent) was directly applied onto the surface of the specimens. Then, the specimens were light-polymerized (Bluephase G2, IvoclarVivadent) with an energy output exceeding $600 \mathrm{mw} / \mathrm{cm}^{2}$ for 120 seconds (each layer of In, By and Ce for 40 seconds) under $250 \mathrm{~g}$ of load (Figure 3). ${ }^{15}$

\section{Color measurements}

Specimens were placed on a white background. ${ }^{16}$ All measurements were recorded in CIELAB coordinates with a spectrophotometer (VITA Easyshade Advance, Vita Zahnfabrik, Bad Sackingen, Germany) by a single experienced operator who had also been trained and calibrated in using the spectrophotometer in order to eliminate inter-examiner reliability. Spectrophotometer (VITA Easyshade) in "Tooth areas" mode (where cervical, middle and incisal areas could be recorded at the same time), determined the values of the CIELAB coordinates for each layer of the ceramic slices [incisal (In), body (Bo) and cervical (Ce)] (Figure 4). The instrument calibration was evaluated before and after measurement of each slice. The tip of the spectrophotometer was placed firmly into the calibration port and was held steadily in place until the instrument sounded a beep to indicate that the calibration was complete. In order to measure the cervical, middle and incisal shades of a tooth, highlighting and selecting the tooth area icon on the measurement menu was followed. The probe tip was placed perpendicular and flush to the surface. The probe on the cervical area of the ceramic-composite specimen was placed and the measurement switch was pressed. Then the body and incisal areas were measured. After three rapid "beeps" a correct measurement was made and "ok" was displayed on the screen. The $\mathrm{L}^{*} \mathrm{a} \mathrm{b}^{*}$ color findings of each specimen was measured 3 times recurrently; an average of the readings was calculated to give the initial color of the specimen.

\section{Statistical analysis}


Statistical analysis was performed using SPSS 18.0 for Windows software (Chicago, IL, USA). The means of each group were analysed by one-way analysis of variance (ANOVA). Shapiro-Wilk test was used to determine the significant differences between each layer when the differences between groups were significant. $\mathrm{P}$ values less than 0.05 were considered to be istatistically significant in all tests.

\section{Results}

Mean $\Delta \mathrm{E}$ values for $\mathrm{A}$ and $\mathrm{B}$ were significant for In $(\mathrm{A}: 6,2 \pm 1,1 ; \mathrm{B}: 6,6 \pm 1,2)$ Bo $(\mathrm{A}: 5,1 \pm 2,6 ; \mathrm{B}: 3,9 \pm 1)$ and $\mathrm{Ce}(\mathrm{A}: 5,3 \pm 2,7 ; \mathrm{B}: 3,8 \pm 1,2)$, respectively . $(\mathrm{p}<0,05)$. While mean $\Delta \mathrm{E}$ values in In for $\mathrm{D}$ and $\mathrm{L}$ for A and B groups were significantly higher $(6,3 \pm 1,2 ; 6,6 \pm 1,2)$ than Bo and Ce for D $(4,4 \pm 2,4 ; 4,6 \pm 2,6)$ and $\mathrm{L}(4,6 \pm 1,4 ; 4,5 \pm 1,7)$ groups $(\mathrm{p}<0.05)$, respectively. Mean differences between Bo and Ce for A $(5,1 \pm 2,6 ; 5,3 \pm 2,7)$ and for $\mathrm{B}(3,9 \pm 1 ; 3,5 \pm 1,2)$ groups were insignificant $(\mathrm{P} \geq 0.05)$ while for In the differences were significant $(6,2 \pm 1,1$ for $\mathrm{A}$ and $6,6 \pm 1,2$ for B) (Table 1).

The values were calculated as $\Delta E$ and the data were statistically analyzed (One-way ANOVA, Shapiro-Wilk). A groups $(0,55 \mathrm{~mm})$ under medium resin cement $(0)$ revealed that mean $\Delta \mathrm{E}$ values In $(5,6 \pm 0,78)$ were significantly higher than $\mathrm{Bo}(2,45 \pm 0,64)$ and $\mathrm{Ce}(2,54 \pm 0,61)$ for light die material groups $(p<0,05)$. Mean differences between In $(6,99 \pm 0,93)$, Bo $(7,72 \pm 0,91)$ and Ce $(8,12 \pm 0,53)$ groups were significant $(p<0,05)$ for the dark die material. A groups $(0,55 \mathrm{~mm})$ under high value resin cement $(+3)$ resulted in mean $\Delta \mathrm{E}$ values in In $(7,25 \pm 0,47)$ that were significantly higher than Bo $(3,56 \pm 0,50)$ and $\mathrm{Ce}(3,13 \pm 0,53)$ for the light die material groups $(p<0,05)$. Mean differences between In $(5,47 \pm .0,95)$, Bo $(6,62 \pm 1,46)$ and Ce $(7,04 \pm .1,05)$ groups were significant $(p<0,05)$ for the dark die material. For A groups $(0,55 \mathrm{~mm})$ under the low value resin cement $(-3)$ mean $\Delta \mathrm{E}$ values in In $(5,24 \pm 0,70)$ were significantly higher than Bo $(2,10 \pm 0,18)$ and Ce $(2,62 \pm 0,62)$ for the light die material groups $(p<0,05)$. Mean differences between In $(7,04 \pm 0,59)$, 
Bo $(8,27 \pm 0,60)$ and $\mathrm{Ce}(8,83 \pm 0,68)$ groups were significant $(p<0,05)$ for the dark die material (Table 1).

For $\mathrm{B}$ groups $(0,80 \mathrm{~mm})$ under the medium resin cement $(0)$; mean $\Delta \mathrm{E}$ values in $\mathrm{In}$ $(7,3 \pm 0,79$. $)$ were significantly higher than Bo $(2,88 \pm 0,95)$ and $\mathrm{Ce}(2,52 \pm .0,23)$ for the light die material (A1) groups $(p<0,05)$. Mean differences between In $(5,42 \pm 0,35)$, Bo $(5,12 \pm 0,82)$ and Ce $(5,21 \pm 0,82)$ groups were significant $(p<0,05)$ for the dark die material $(\mathrm{A} 3,5)$. B groups $(0,80 \mathrm{~mm})$ under the high value resin cement $(+3)$ exhibited mean $\Delta \mathrm{E}$ values in $\mathrm{In}$ as $8,19 \pm 0,54$ that were significantly higher than Bo $(3,75 \pm 0,46)$ and $\mathrm{Ce}(3,25 \pm .0,39)$ for the light die material (A1) groups $(p<0,05)$. Mean differences between In $(5,79 \pm .0,18$.$) , Bo (4,46 \pm 0,60)$ and Ce $(4,48 \pm 0,87)$ groups were significant $(p<0,05)$ for the dark die material. For B groups $(0,80 \mathrm{~mm})$ under the low value resin cement $(-3)$; mean $\Delta \mathrm{E}$ values in In $(7,85 \pm 0,69)$ were significantly higher than Bo $(2,87 \pm 0,61)$ and $\mathrm{Ce}(2,35 \pm 0.31)$ for the light die material groups $(p<0,05)$. Mean differences between In $(5,27 \pm 0,32)$, Bo $(4,38 \pm 0,46)$ and $\mathrm{Ce}(4,71 \pm 0,46)$ groups were significant $(p<0,05)$ for the dark die material (Table 1) (Graphics 1,2).

\section{Discussion}

In this study, the effect of cement color, die color and ceramic thickness on the post-cementation color of thin and ultra-thin laminate veneers milled from multilayered glass-ceramic blocks for each layer was evaluated. Final color changes of ceramic specimens that were polymerized beneath two different ceramic thicknesses and two different die colors after adhesive cementation were observed. There were significant differences in color changes within groups. The differences within body and cervical groups were insignificant, while significant differences were observed between incisal and and other groups (body (Bo)-cervical (Ce)). The thickness of the ceramic and die color 
also affected the final color of laminate veneers which were fabricated from a multilayered block. Therefore, the null hypothesis was rejected.

The magnitude of $\Delta \mathrm{E}$ units was used in this study to demonstrate the clinical effect of ceramic veneer thickness and the resin cements used on the final shade of the specimens. The study adopted the value of $\Delta \mathrm{E}>3$ as an unacceptable color difference. ${ }^{14}$

Previously introduced CAD/CAM ceramic blocks were monochromatic. With the proposal of recently introduced polychromatic blocks having multi layers imitating cervical, dentin and enamel; chairside applications have been more improved for the dentist to finalize all clinical steps of a ceramic restoration in a single appointment. This has been obtained through incorporation of colour pigments present in the framework blocks into the gradient chromatic ceramic blocks. ${ }^{6,17}$ However, besides their above mentioned advantage, the achievement of the expected final colour of a restoration is questionable since no studies exist on how and in which way the developed digital systems and the various framework ceramic blocks do influence the esthetic outcome.

A conservative preparation with a minimal tooth reduction, esthetics, and maintenance of healthy tissues are the major advantages of ceramic laminate veneers. Since ceramic is a translucent material, tooth-coloured resin cement under these restorations is mainly reflected from beneath the restoration for optimal esthetics. ${ }^{18}$ On the other hand, this might be a disadvantage in situations with unpredictable post-cementation color with ultra-thin ceramic veneer restorations. ${ }^{1}$ Even when adequate ceramic thicknesses are simulated, clinical shade matches are difficult to achieve especially on a dark substructure. ${ }^{19}$ To overcome this problem, shade variety has been increased in newly introduced resin luting materials so that some resin cements might mask the dark-colored teeth where the final color of the resoration may be compromised. ${ }^{18}$

Resin luting cements may be chemically, photo or dual-polymerized. ${ }^{20}$ The chemical composition and colour of a luting cement may affect the esthetic appearance, especially when used 
with ultra-thin translucent ceramic veneers. ${ }^{21}$ The oxidation of reactive groups with amine accelerators and inhibitors in the course of chemical or dual-curing, may cause color changes. The quality of polymerization also influences discoloration since the amount of residual monomers lead to formation of coloured degradation products. ${ }^{10}$ Some photo-and dual-polymerized resin cements contain camphorquinone as a photo initiator, which has an intense yellow color that remains also yellow after photo activation. ${ }^{22}$ It has been previously reported that the saffron present in the content of camphorquinone was effective on the post-cementation colour. ${ }^{23}$ Therefore, in order to maintain the esthetic outcome for a long period, phenylpropanedione (PPD;1-phenyl-1,2propanedione) or Lucirin ${ }^{\circledR}$ (2,4,6-trimethyl- benzoyldiphenylphosphine oxide) containing resin cements have been adopted since they are completely colorless after light-polymerization and its polymers are less yellowish. ${ }^{24}$ The base paste of the resin luting cement used in this study contained both aliphatic and aromatic tertiary amine and the catalyst paste benzoyl peroxide. The color changes of a dual-curing luting resin are mainly caused by the oxidization of the amine; a necessary component as a polymerization initiator; maintained by the catalyst paste. ${ }^{25}$ Since no aging procedures were performed in the present study to reduce the parameters affecting on the multicoloured ceramic block specimens, no additional negative yellowish contributing factor for discoloration of the underlying resin luting cement was observed. However, this parameter should also be considered for further studies after aging.

Optical properties of a ceramic material are influenced by the microstructure and composition of the material. Since color and other optical features such as scattering and absoption of light are important for shade matching and esthetic outcome, effects of these have also been proposed for further inclusion parameters for studies on colour. ${ }^{14}$ The present study focused on the effect of multilayered ceramic blocks on final color of veneer-simulating specimens after luting through the most commonly measured parameter; tranclucency (TP), where it was defined as the color difference $(\Delta \mathrm{E})$ between a material of uniform thickness over a white (light) and a black 
(dark) background. ${ }^{26}$ Therefore, a flat surface die and uniform thickness veneer specimens from cervical to incisal thirds were fabricated in order to eliminate the above mentioned plane related optical varieties.

In the present study, when different thickness ceramics with different backgrounds and cements were evaluated on the light die material using multilayered blocks; significantly higher $\Delta \mathrm{E}$ values were obtained for the incisal area when compared with the middle (body) and cervical regions. This was attributed to the lower colour pigment ingredient of the incisal layer. This may cause inconsistencies in colour of restorations fabricated from multilayered blocks after cementation in practice. Furthermore, a cement line may become visible at the incisal area.

The present study revealed significant $\Delta \mathrm{E}$ values for all three layers for measurements made on the dark die material for both ceramic thicknesses, although the colour differences among layers were similar. For all cement types, the obtained values for $0,55 \mathrm{~mm}$ thick specimens were significantly higher than the ones with $0,80 \mathrm{~mm}$ thickness. However, while $\Delta \mathrm{E}$ values for $0,80 \mathrm{~mm}$ thick ceramics were relatively low, it was observed that they were unable to mask the framework colour and the dark substrate colour was reflected to the final shade. This situation could be explained by the thickness of the ceramic veneers applied on the dark die material as well as the ceramic and cement colour. The clinical reflection of this condition may manifest itself as a problem when the tooth colour shine through is try to be changed by the colour of the cement and the ceramic veneer for a dark substrate shade. In such cases, low translucent monochromatic spesific blocks can be chosen instead of multilayered blocks. Besides, medium thickness $(0.80 \mathrm{~mm}$ or higher) ceramic veneers may also obscure the framework colour. It is impossible to mask a strong discoloration by a thin layer of ceramic $(0.3-0.7 \mathrm{~mm})$ without making the restoration opaque and dull. Consequently, the restored tooth would never have the same translucency as the adjacent natural teeth. ${ }^{27}$ 
The obtained values for the three cements for both thicknesses and die materials were found to be significant in this study. The $\Delta \mathrm{E}$ values fort he medium translucency cement group were higher than the +3 and -3 cement group values. This might have stemmed from the translucent character of the medium opacity cement colour thus not exhibiting any post-cementation colour change, but the effect of the colour pigments in the +3 and -3 cement groups on the final shade. Some failures in reaching the desired final colour with the selected cement shades for various substrate gradients,may occur in clinical practice. As the ceramic material thickness applied on a light die shade is increased because of the incisal translucency, the $\Delta \mathrm{E}$ values were concluded to increase as well,. This means that as the thickness increases, one cannot take advantage of the underlying cement colour.

One of the limitations of the present study was using only one shade of multilayered block (A2) with three luting resin cement types. Further studies should be conducted considering the other shades of luting cements .

\section{Conclusion}

Within the limitations of this study the following conclusions were made:

1. Colour differences on the incisal area were higher with respect to the middle and incisal regions when multilayered blocks were used on a light coloured framework.

2. Colour mismatch or reflection of the cement line in the incisal third may occur in thin veneers obtained from multilayered blocks after cementation. 
3. The $\Delta \mathrm{E}$ values for thin veneers for the three cements on the dark framework were higher than the values obtained from thick specimens; referring to the preference of monochromatic blocks with lower translucency in terms of clinical relevance.

4. Ceramic veneers with at least $0,80 \mathrm{~mm}$ thickness or more may mask the substrate colour.

\section{References}

1. Ozturk E, Hickel R, Bolay S, et al. Micromechanical properties of veneer luting resins after curing through ceramics. Clin Oral Invest 2012;16:139-146

2. Volpato CA, Monteiro S Jr, de Andrada MC, et al. Optical influence of the type of illuminant, substrates and thickness of ceramic materials. Dent Mater 2009;25:87-93. 
3. Motro PF, Kursoglu P, Kazazoglu E. Effects of different surface treatments on stainability of ceramics. J Prosthet Dent 2012;108:231-237

4. Liu PR, Essig ME. Panorama of dental CAD/CAM restorative systems. Compend Contin Educ Dent 2008;29:482, 484, 486-488

5. Conrad HJ, Seong WJ, Pesun IJ. Current ceramic materials and systems with clinical recommendations: a systematic review. J Prosthet Dent 2007;98:389-404

6. Reich S, Hornberger H. The effect of multicolored machinable ceramics on the esthetics of all-ceramic crowns. J Prosthet Dent 2002;88:44-49

7. Shenoy A. Survival rates of porcelain laminate restoration based on different incisal preparation designs: An analysis. J Conserv Dent 2011;14:203

8. Turgut S, Bagis B. Effect of resin cement and ceramic thickness on final color of laminate veneers: an in vitro study. J Prosthet Dent 2013;109:179-186

9. Nosti J. "Thin is in" the art of minimal \& no prep veneer. J N J Dent Assoc 2009;80:30-31

10. Turgut S, Bagis B. Colour stability of laminate veneers: an in vitro study. J Dent 2011;39:57-64

11. Kucukesmen HC, Usumez A, Ozturk N, et al. Change of shade by light polymerization in a resin cement polymerized beneath a ceramic restoration. J Dent 2008;36:219-223

12. Lee $\mathrm{YK}, \mathrm{Yu} \mathrm{B}$, Lee $\mathrm{SH}$, et al. Variation in instrument-based color coordinates of esthetic restorative materials by measurement method-A review. Dent Mater 2010;26:1098-1105

13. Ozturk O, Uludag B, Usumez A, et al. The effect of ceramic thickness and number of firings on the color of two all-ceramic systems. J Prosthet Dent 2008;100:99-106

14. Della Bona A, Nogueira AD, Pecho OE. Optical properties of CAD-CAM ceramic systems. J Dent 2014;42:1202-1209

15. Wang CJ, Millstein PL, Nathanson D. Effects of cement, cement space, marginal design, seating aid materials, and seating force on crown cementation. J Prosthet Dent 1992;67:786-790 
16. Zenthöfer A, Cabrera T, Corcodel N, Rammelsberg P, Hassel AJ. Comparison of the Easyshade Compact and Advance in vitro and in vivo. Clin Oral Investig 2014;18:1473-1479

17. Yuan K, Wang F, Gao J, et al. Effect of zircon-based tricolor pigments on the color, microstructure, flexural strength and translucency of a novel dental lithium disilicate glass-ceramic. J Biomed Mater Res B Appl Biomater 2014;102:98-107

18. Kesrak P, Leevailoj C. Surface Hardness of Resin Cement Polymerized under Different Ceramic Materials. Int J Dent 2012;2012:317-509

19. Cho MS, Yu B, Lee YK. Opalescence of all-ceramic core and veneer materials. Dent Mater 2009;25:695-702

20. Rosenstiel SF, Land MF, Crispin BJ. Dental luting agents: A review of the current literature. J Prosthet Dent 1998;80:280-301

21. Pazin MC, Moraes RR, Gonçalves LS, et al. Effect of ceramic thickness and curing unit on light transmission through leucite-reinforced material and polymerizatiın of dual-cured luting agent. J Oral Sci 2008;50:131-136

22. Park YJ, Chae KH, Rawls HR. Development of a new photoinitiation system for dental light-cure composite resins. Dent Mater 1999;15:120-127

23. Omar H, Atta O, El-Mowafy O, et al. Effect of CAD-CAM porcelain veneers thickness on their cemented color. J Dent 2010;38:95-99

24. Porto ICCM, Soares LES, Martin AA, et al. Influence of the photoinitiator system and light photoactivation units on the degree of conversion of dental composites. Braz Oral Res 2010;24:475481

25. Manso AP, Silva NR, Bonfante EA, et al. Cements and adhesives for all-ceramic restorations. Dent Clin North Am 2011;55:311-332

26. Barizon KTL, Bergeron C, Vargas MA, et al. Ceramic materials for porcelain veneers: Part II. Effect of material, shade, and thickness on translucency. J Prosthet Dent 2014;112:864-870 
27. Peumans M, Van Meerbeek B, Lambrechts P, et al. Porcelain veneers: a review of the literature. J Dent 2000;28:163-177

\section{Legends}

Figure 1 Sectioning of the ceramic slices for different thicknesses.

Figure 2 Preparation of the resin die specimens in different shades. 
Figure 3 Cementation of the ceramic veneers onto the die materials.

Figures 4a-c Colour measurement with the dental spectrophotometer at a) cervical, b) middle and c) incisal areas of the specimens.

Table 1 The mean $\Delta \mathrm{E}$ values and levels of significance according to differing ceramic thicknesses, cements and framework colour ( $P \leq 0.05$ MT: medium value (C group), HT: high value $\Delta \mathrm{E}$ cy (L group), $L T$ : low value (D group) )

Graphic 1 Mean $\Delta E$ values for $0,55 \mathrm{~mm}$ thick specimens with regard to resin cement colour (D, C, L) and three regions (Ce:cervical, Bo: Body, In: Incisal)

Graphic 2 Mean $\Delta E$ values for $0,8 \mathrm{~mm}$ thick specimens with regard to resin cement colour (D, C, L) and three regions (Ce:cervical, Bo: Body, In: Incisal) 


\section{Page 17 of 24} Journal of Prosthodontics

1

2

3

4

5

6

7

8

9

10

11

12

14

15

16

17

18

19

20

21

22

23

24

25

26

27

28

29

30

31

32

33

34

35

36

37

38

39

40

41

42

43

44

45

46

47

48

49

50

51

52

53

54

55

56

57

58

59

60

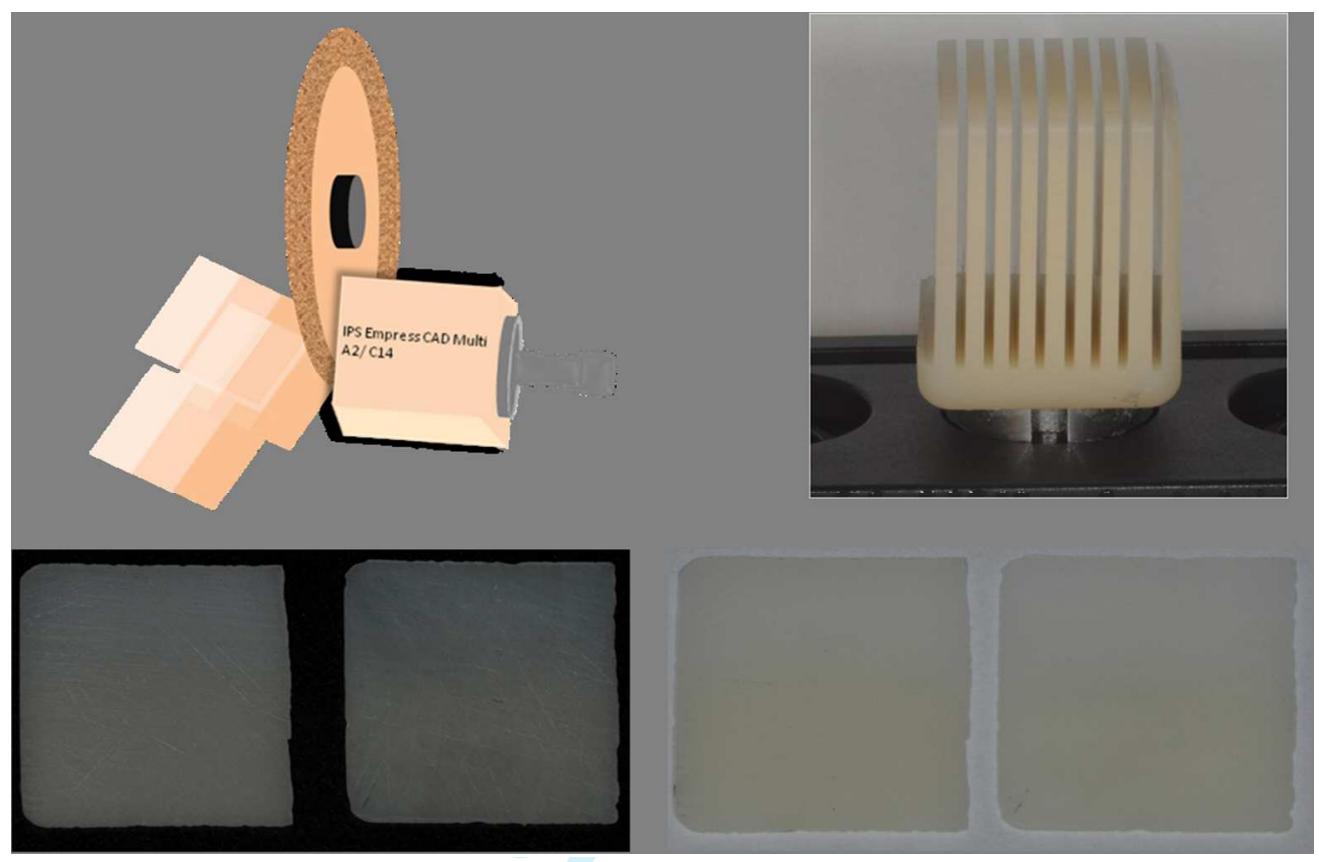




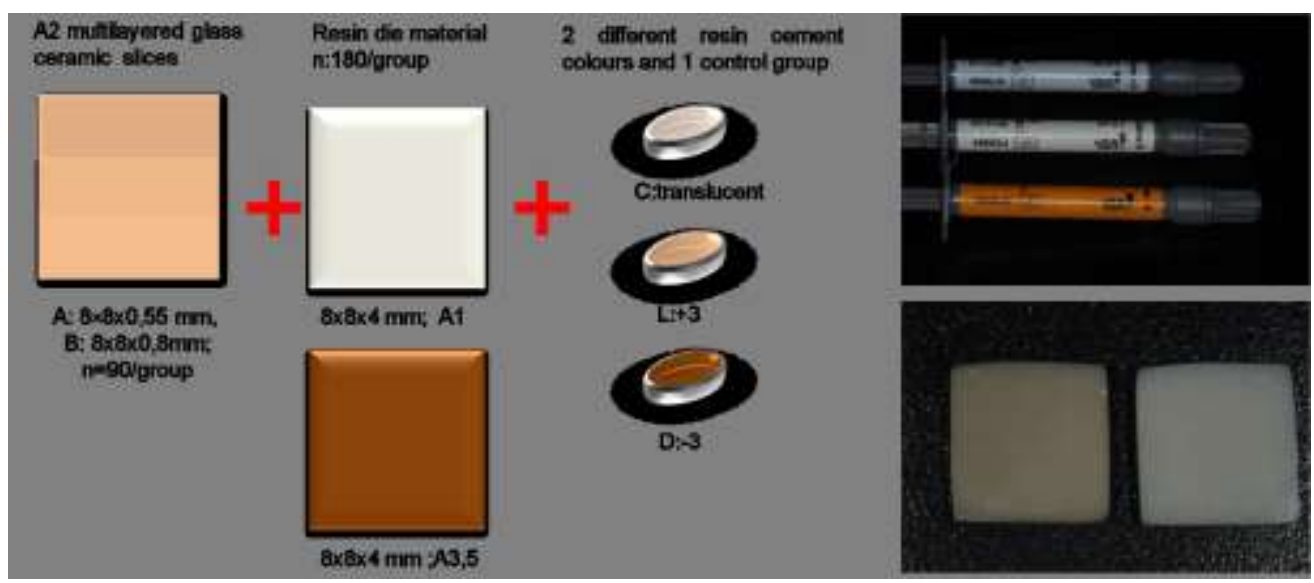




\section{Page 19 of 24}

Journal of Prosthodontics

1
2
3
4
5
6
7
8
9
10
11
12
13
14
15
16
17
18
19
20
21
22
23
24
25
26
27
28
29
30
31
32
33
34
35
36
37
38
39
40
41
42
43
44
45
46
47
48
50
59
50
52
53
50

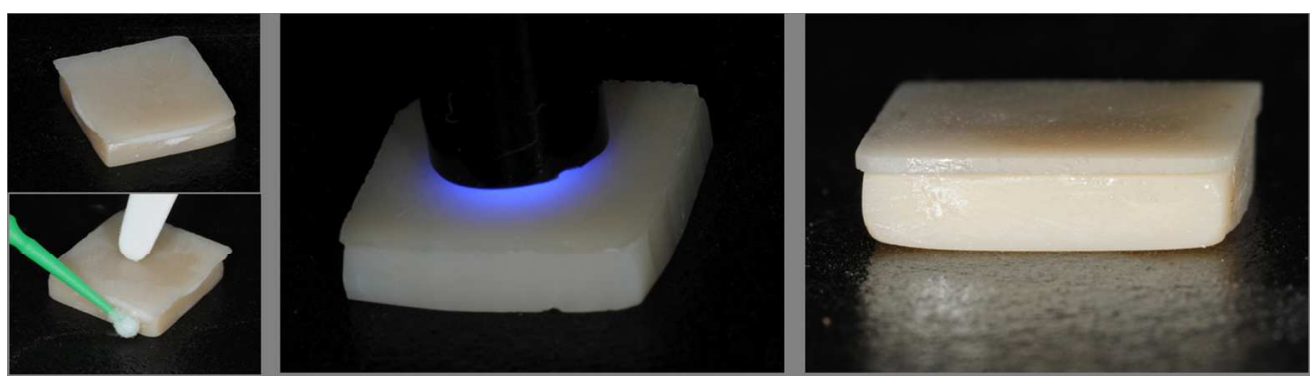

16

17

19

20

21

23

24

25

26

27
28

29

30

31

33

34

35

36
37

38

39

40

41

42

43
44

45

46

47

48

49

50

52

53

54

55

56 


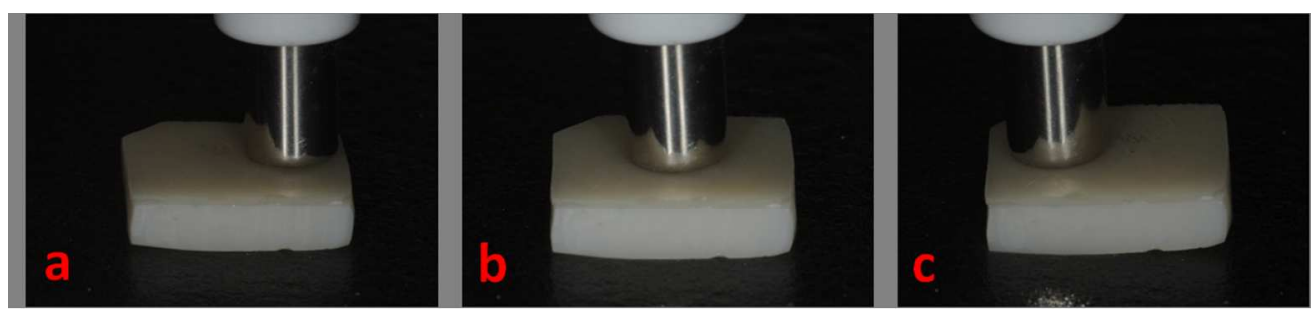




\begin{tabular}{|c|c|c|c|c|c|c|c|}
\hline \multicolumn{8}{|c|}{ Thickness / 0.55} \\
\hline \multicolumn{4}{|c|}{ Substracture (light) } & \multicolumn{4}{|c|}{ Substracture (dark) } \\
\hline & Cement & Cement & $\mathrm{P}$ & & Cement & Cement & $\mathrm{P}$ \\
\hline \multirow{6}{*}{$\Delta \mathrm{Ce} .}$. & \multirow{2}{*}{$\mathrm{MT}$} & $\mathrm{HT}$ & _ & \multirow{6}{*}{$\Delta \mathrm{Ce} .}$. & \multirow{2}{*}{$\mathrm{MT}$} & $\mathrm{HT}$ & 0.014 \\
\hline & & $\mathrm{LT}$ & _ & & & $\mathrm{LT}$ & _ \\
\hline & \multirow{2}{*}{$\mathrm{HT}$} & $\mathrm{MT}$ & - & & \multirow{2}{*}{$\mathrm{HT}$} & $\mathrm{MT}$ & 0.014 \\
\hline & & $\mathrm{LT}$ & _ & & & $\mathrm{LT}$ & 0.000 \\
\hline & \multirow{2}{*}{$\mathrm{LT}$} & $\mathrm{MT}$ & _ & & \multirow{2}{*}{$\mathrm{LT}$} & $\mathrm{MT}$ & _ \\
\hline & & $\mathrm{HT}$ & - & & & $\mathrm{HT}$ & 0.000 \\
\hline \multirow{6}{*}{$\Delta$ Bo.. } & \multirow{2}{*}{$\mathrm{MT}$} & $\mathrm{HT}$ & 0.000 & \multirow{6}{*}{$\Delta$ Bo.. } & \multirow{2}{*}{$\mathrm{MT}$} & $\mathrm{HT}$ & _ \\
\hline & & $\mathrm{LT}$ & _ & & & $\mathrm{LT}$ & - \\
\hline & \multirow{2}{*}{$\mathrm{HT}$} & $\mathrm{MT}$ & 0.000 & & \multirow{2}{*}{$\mathrm{HT}$} & $\mathrm{MT}$ & - \\
\hline & & $\mathrm{LT}$ & 0.000 & & & $\mathrm{LT}$ & 0.005 \\
\hline & \multirow{2}{*}{$\mathrm{LT}$} & $\mathrm{MT}$ & - & & \multirow{2}{*}{$\mathrm{LT}$} & $\mathrm{MT}$ & - \\
\hline & & $\mathrm{HT}$ & 0.000 & & & $\mathrm{HT}$ & 0.005 \\
\hline \multirow{6}{*}{$\Delta \ln .}$. & \multirow{2}{*}{$\mathrm{MT}$} & $\mathrm{HT}$ & 0.000 & \multirow{6}{*}{$\Delta \ln .}$. & \multirow{2}{*}{$\mathrm{MT}$} & $\mathrm{HT}$ & 0.001 \\
\hline & & $\mathrm{LT}$ & _ & & & $\mathrm{LT}$ & - \\
\hline & \multirow{2}{*}{$\mathrm{HT}$} & $\mathrm{MT}$ & 0.000 & & \multirow{2}{*}{$\mathrm{HT}$} & $\mathrm{MT}$ & 0.001 \\
\hline & & $\mathrm{LT}$ & 0.000 & & & $\mathrm{LT}$ & 0.001 \\
\hline & \multirow{2}{*}{$\mathrm{LT}$} & $\mathrm{MT}$ & - & & \multirow{2}{*}{$\mathrm{LT}$} & $\mathrm{MT}$ & - \\
\hline & & $\mathrm{HT}$ & 0.000 & & & $\mathrm{HT}$ & 0.001 \\
\hline
\end{tabular}




\begin{tabular}{|c|c|c|c|c|c|c|c|}
\hline \multicolumn{8}{|c|}{ Thickness / 0.80} \\
\hline \multicolumn{4}{|c|}{ Substracture (light) } & \multicolumn{4}{|c|}{ Substracture (dark) } \\
\hline & Cement & Cement & $\mathrm{P}$ & & Cement & Cement & $\mathrm{P}$ \\
\hline \multirow{6}{*}{$\Delta$ Ce.. } & \multirow{2}{*}{ M T } & H T & 0.000 & \multirow{6}{*}{$\Delta \mathrm{Ce}}$. & \multirow{2}{*}{ M T } & H T & _- \\
\hline & & LT & _ & & & LT & _ \\
\hline & \multirow{2}{*}{$\mathrm{HT}$} & $\mathrm{MT}$ & 0.000 & & \multirow{2}{*}{ H T } & $\mathrm{MT}$ & _ \\
\hline & & LT & 0.000 & & & LT & _- \\
\hline & \multirow{2}{*}{ LT } & $\mathrm{MT}$ & - & & \multirow{2}{*}{ LT } & $\mathrm{MT}$ & - \\
\hline & & H T & 0.000 & & & H T & _ \\
\hline \multirow{6}{*}{$\Delta$ Bo.. } & \multirow{2}{*}{ M T } & H T & 0.032 & \multirow{6}{*}{$\Delta$ Bo.. } & \multirow{2}{*}{$\mathrm{MT}$} & $\mathrm{HT}$ & _- \\
\hline & & LT & _ & & & LT & _ \\
\hline & \multirow{2}{*}{$\mathrm{HT}$} & $\mathrm{MT}$ & 0.032 & & \multirow{2}{*}{ H T } & $\mathrm{MT}$ & - \\
\hline & & LT & 0.030 & & & LT & - \\
\hline & \multirow{2}{*}{$\mathrm{LT}$} & M T & _- & & \multirow{2}{*}{ LT } & M T & _- \\
\hline & & H T & 0.030 & & & $\mathrm{HT}$ & - \\
\hline \multirow{6}{*}{$\Delta \ln .}$. & \multirow{2}{*}{ M T } & H T & 0.022 & \multirow{6}{*}{$\Delta \ln }$. & \multirow{2}{*}{ M T } & $\mathrm{HT}$ & 0.031 \\
\hline & & LT & - & & & LT & - \\
\hline & \multirow{2}{*}{ H T } & M T & 0.022 & & \multirow{2}{*}{ H T } & M T & 0.031 \\
\hline & & LT & _ & & & LT & 0.002 \\
\hline & \multirow{2}{*}{ LT } & M T & _- & & \multirow{2}{*}{ LT } & $\mathrm{MT}$ & _ \\
\hline & & $\mathrm{HT}$ & _- & & & $\mathrm{HT}$ & 0.002 \\
\hline
\end{tabular}




\section{Page 23 of 24}

Journal of Prosthodontics

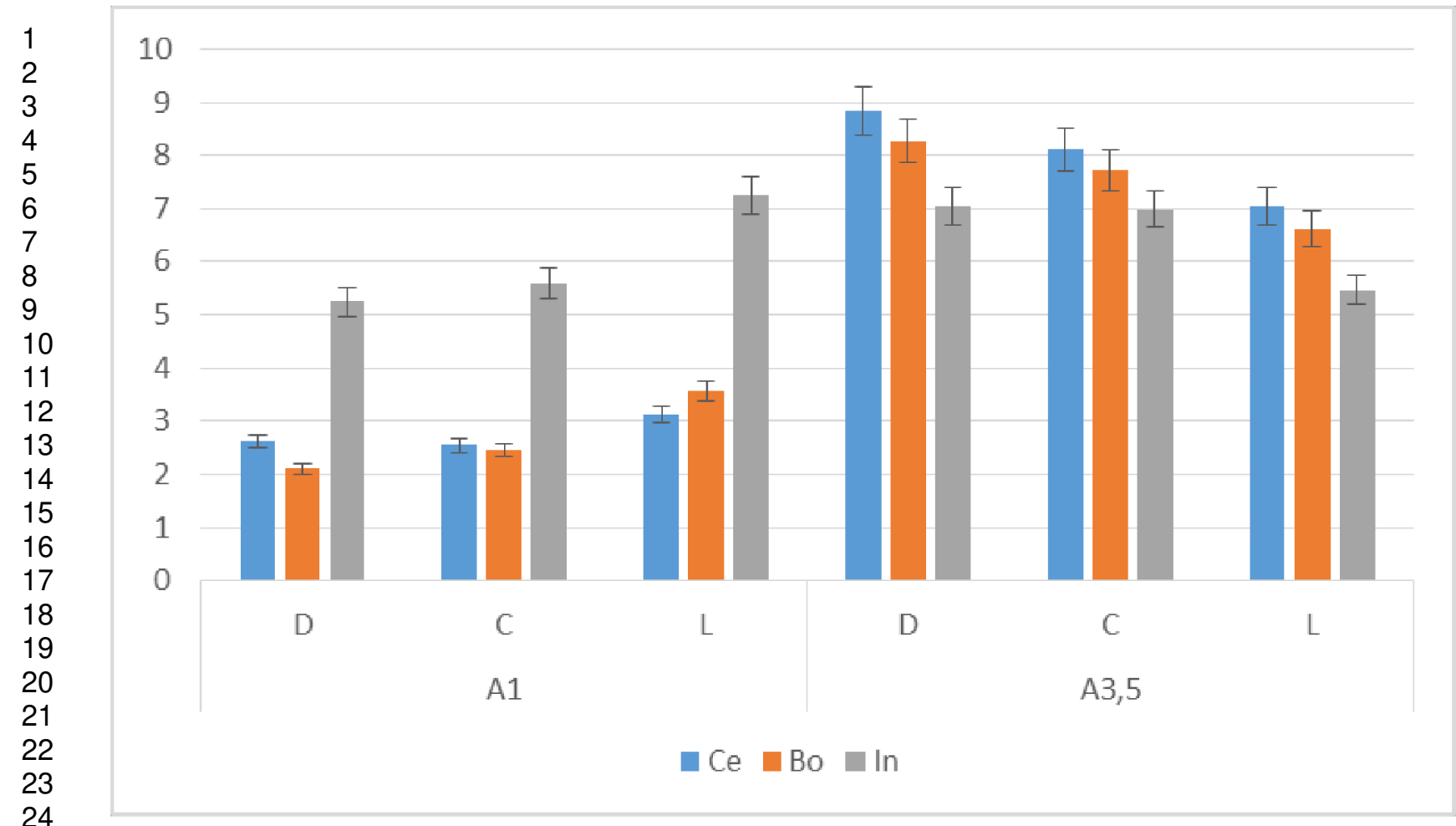

$\Delta$

Journal of Prosthodontics 


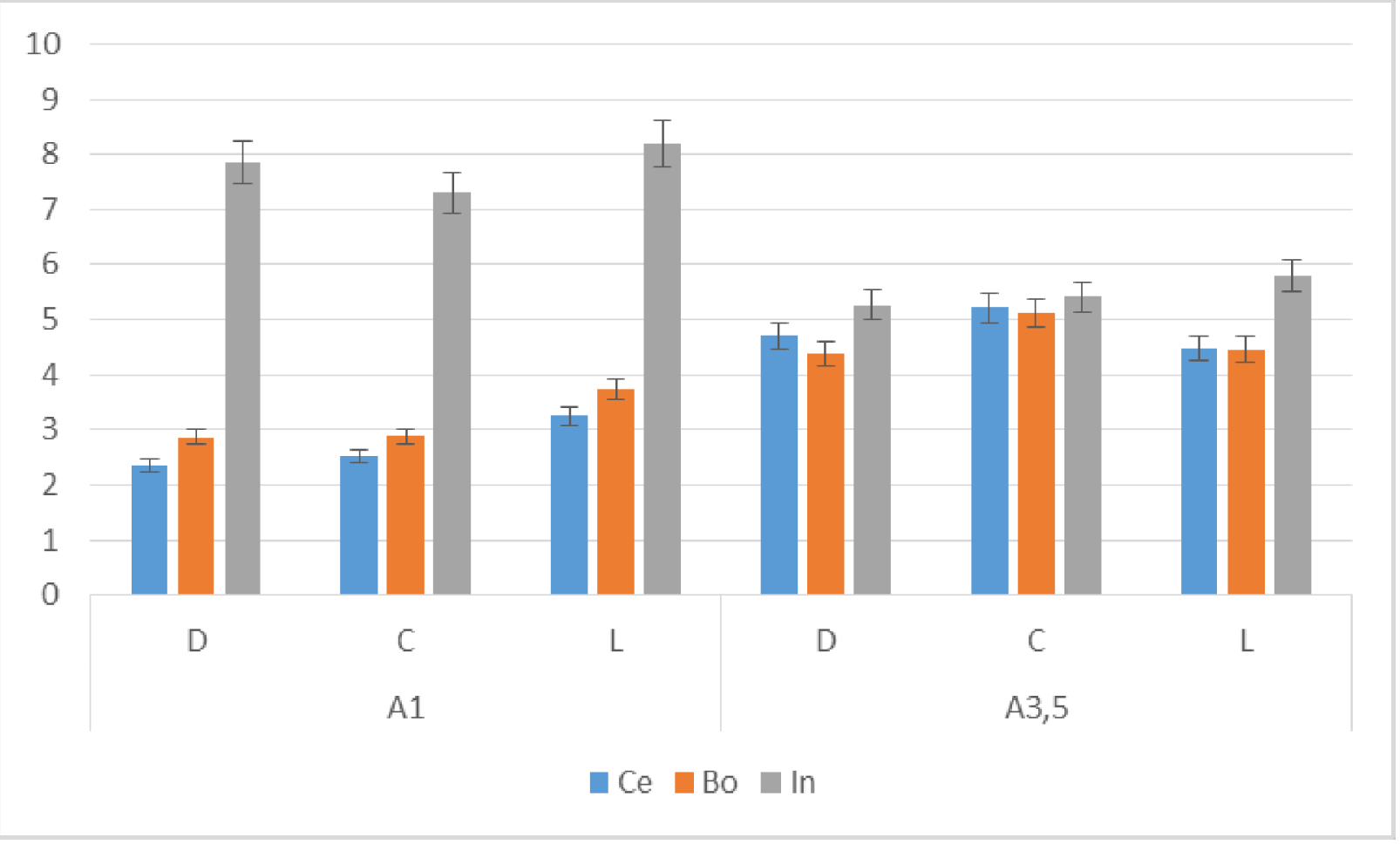

$\Delta$

Journal of Prosthodontics 